\title{
Development of a constitutive model for DPX2 explosive
}

\author{
Philip Church ${ }^{1}$, Peter Gould ${ }^{2 *}$, and David Williamson ${ }^{3}$ \\ ${ }^{1}$ QinetiQ, Fort Halstead, Sevenoaks, Kent, TN14 7BP, UK \\ ${ }^{2}$ QinetiQ, Bristol Business Park, Coldharbour Lane, Bristol, BS16 1FJ, UK \\ ${ }^{3}$ Surface, Microstructure \& Fracture Group, Cavendish Laboratory, JJ Thomson Ave, Cambridge, CB3 0HE, UK.
}

\begin{abstract}
There is a significant challenge in simulating the behaviour of PBXs under high strain rate impact loading. A Porter-Gould physically based constitutive model has been developed for the DPX2 explosive. A series of quasi-static compression and tensile tests over a range of temperatures were performed together with DMA tests to calibrate the model. In particular tests were performed for different $\mathrm{L} / \mathrm{D}$ ratios to understand the complex localisation and damage behaviour of the material. High rate tests on the compression Split Hopkinson Pressure Bar (SHPB) for a range of temperatures were then used for validation of the model under idealised stress states. Some model development is still required, particularly at lower temperatures near the glass transition temperature. In addition a series of classical Taylor Tests were used to validate the model under impact loading conditions at room temperature. The DYNA3D simulations gave very good results compared to the experiments for these impact conditions.
\end{abstract}

\section{Introduction}

The UK Ministry of Defence has a desire to move to explosives that have both higher performance and lower sensitiveness than historic compositions and this has led them to consider DPX2 as a candidate.

DPX2 is a pressed explosive which has a higher HMX content than the explosives that it is designed to replace and should thus have increased performance. There is, however, very little information on its mechanical performance - a key criterion in its response to environmental stimuli and in Hazard scenarios. Data that exist on PBXN5 - a similar composition - suggest that the mechanical response will be dominated by shear failure [1] and this is borne out by the observed fracture response, Figures 1 and 2. Such fracture behaviour is often seen in granular materials and highly loaded particulate composites. In order to address this lack a comprehensive test regime was proposed covering physical and mechanical properties of DPX2 over a range of strain rates and temperatures. These data would then be used to generate a numerical simulation capability to allow design and assessment of warhead concepts.

\section{Material}

DPX2 Type 1 was procured from Chemring Nobel AS in the form of moulding powder $-2 \mathrm{~mm}$ diameter white spherical particles - and pressed at QinetiQ Fort Halstead to a density of $1760 \mathrm{~kg} \mathrm{~m}^{-3}$. It is $92 \%$ by mass HMX with a HyTemp binder plasticised with DOA. Mechanical test specimens were machined from large billets to the relevant size.

\section{Experiments}

The experiments consisted of:

- Dynamic Mechanical Analysis (DMA),

- Tension and compression at low rate,

- Split Hopkinson Pressure Bar (SHPB),

- Taylor tests at various impact speeds.

All tests apart from the Taylor tests were carried out at various temperatures; these temperatures were determined by analysis of the DMA output. The compression testing at low rate also examined the effect of specimen size and aspect ratio. The SHPB test results were used as verification of the numerical simulations as well as for characterisation of the material model. The numerical simulation capability was then validated by comparison to the Taylor test output.

DMA carried out at $1 \mathrm{~Hz}$ gives the loss tangent for DPX2 presented in Figure 3. It can be seen from this Figure that there will be little change in the properties of the material above room temperature (at $1 \mathrm{~Hz}$ ) so testing 
focussed on lower rate-equivalent temperatures. In determining those rates it was presumed that the shift factor would be approximately $15 \mathrm{~K}$ per decade of rate. So low rate testing was carried out at $190 \mathrm{~K}$ (the start of the second upturn in loss) and $235 \mathrm{~K}$ (to split between the low temperature and room temperature) as well as room temperature. High rate testing was carried out at intervals of $10 \mathrm{~K}$ from $250 \mathrm{~K}$ to $320 \mathrm{~K}$.

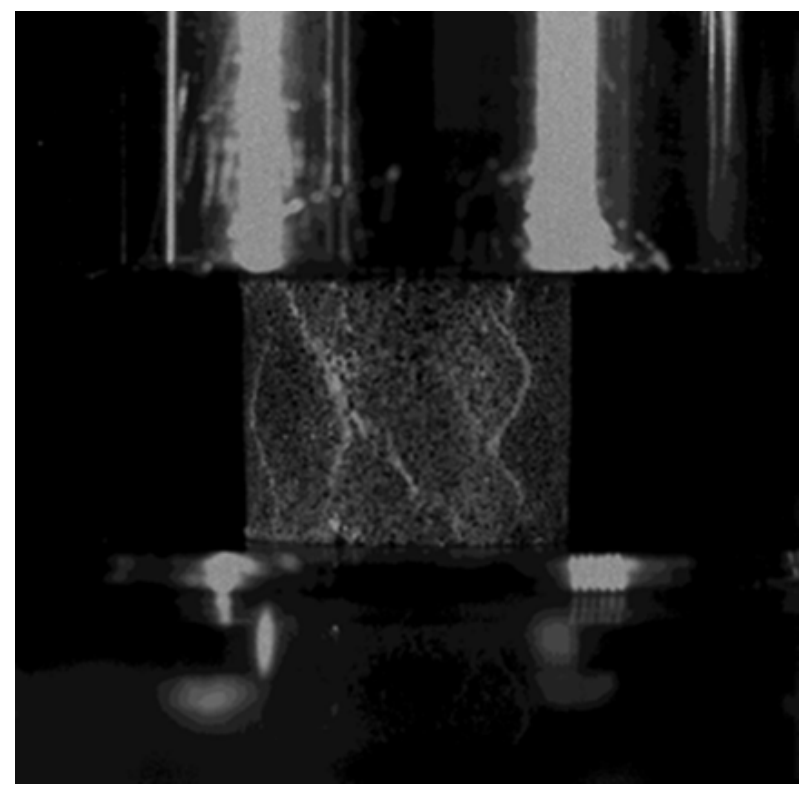

Fig. 1. Fracture of a low aspect ratio specimen at low rate and at room temperature.

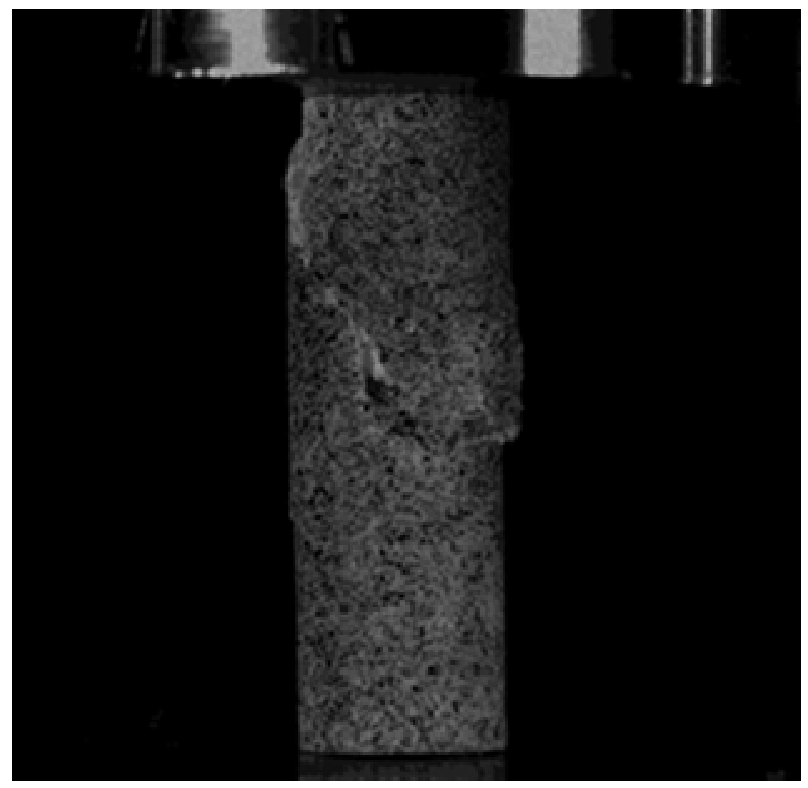

Fig. 2. Fracture of high aspect ratio specimen at low rate and at room temperature.

Nomenclature for compression test samples takes the form "diameter"b"length". So "7b24.5" is a sample with a diameter of $7 \mathrm{~mm}$ and a length of $24.5 \mathrm{~mm}$ giving an aspect ratio of 3.5 .

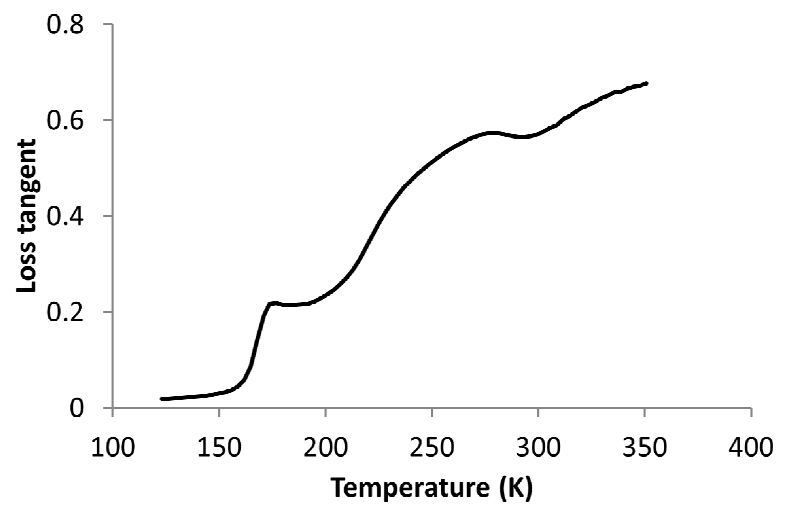

Fig. 3. Loss tangent of DPX2 as a function of temperature at 1 Hz.

All low strain-rate compression experiments were performed using a screw-driven Instron 5500 equipped with a $2 \mathrm{kN}$ load cell. Samples were compressed between surface-ground stainless-steel anvils and were lubricated with soft paraffin wax. Strain was measured using a clip-gauge extensometer (Instron model 2630102) mounted across the anvils. Sample deformation was additionally visualised during the experiments using a Phantom V4.3 video camera. Two repeat experiments were conducted per condition using cylindrical specimens. In each case the true strain is plotted against a value of true stress obtained by making the common assumption that any deformation will conserve volume. This assumption is not likely to lead to significant error at low rate due to the very low fracture strains which imply only minor amounts of particle debonding. In all tests temperature was controlled by passing gaseous nitrogen through a heat exchanger immersed in liquid nitrogen and having that temperature conditioned gas flood the environmental chamber around the specimen. Temperature was measured using K-type thermocouples mounted on each anvil and experiments were conducted only after having held at the target temperature for not less than 30 minutes.

The tensile tests were performed using standard JANNAF samples with a $10 \mathrm{~mm}$ square cross-section. Strain was measured over a $20 \mathrm{~mm}$ gauge length using a video strain extensometer.

All high strain-rate experiments were performed using a magnesium alloy split-Hokinson pressure bar. The bars were nominally $12.7 \mathrm{~mm}$ in diameter and both input and output bars were $500 \mathrm{~mm}$ long. Samples were lubricated with soft paraffin wax. The bars were instrumented with Kulite semiconductor products strain gauges (model AFP 500-090) in a potential divider configuration and positioned midway along the input and output bars. Samples were nominally $6 \mathrm{~mm}$ in diameter and $3 \mathrm{~mm}$ in thickness. In each case the measured strainrate was nominally $1600 \mathrm{~s}^{-1}$.

For the Taylor tests specimens nominally $9 \mathrm{~mm}$ in diameter and $22.5 \mathrm{~mm}$ in length were fired unsaboted from a smooth bore gas-gun towards a large stainlesssteel mass (circa. $60 \mathrm{~kg}$ ) with a surface ground finish. The impact surface was lubricated with soft paraffin 
wax. The impacts were arranged to occur normal to the anvil surface. These experiments were conducted at room temperature. The impacts were filmed using a Phantom v610 high-speed digital video camera.

\section{Results}

A comparison between tension and compression is given in Figure 4. The cross-sectional areas of the specimens are similar. It can be seen that the initial modulus is the same but that fracture occurs at significantly lower strain in tension. The shape of both curves is typical of these materials.

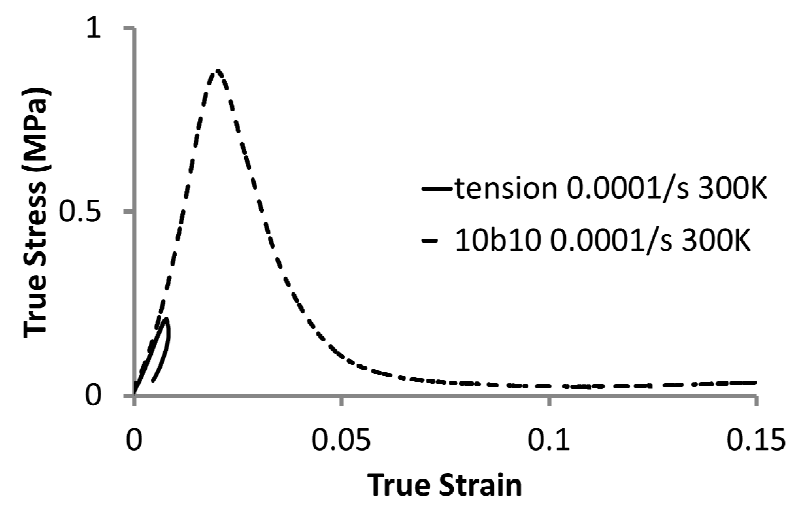

Fig. 4. Comparison between tension and compression response of DPX2 at room temperature and low rate.

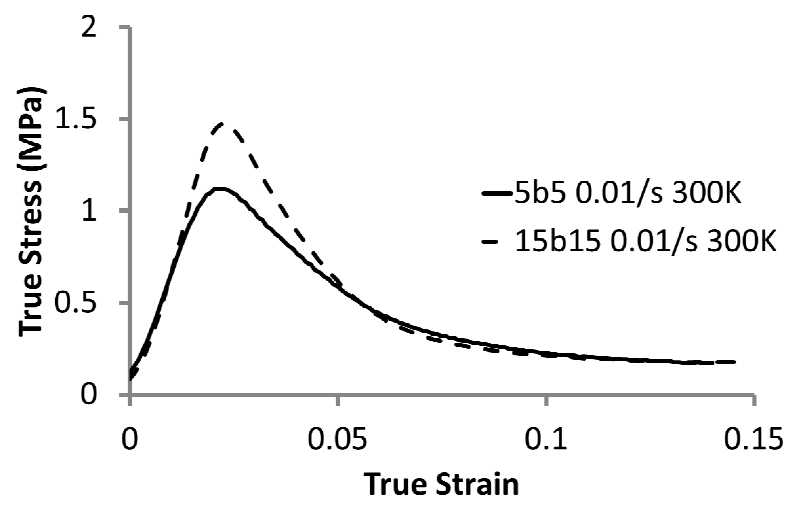

Fig. 5. Effect of sample size at room temperature and low rate.

It can be seen that the size, holding aspect ratio constant, of samples has some effect at low rate. Figure 5 shows the extremes of results: other intermediate sized samples fell between the two curves shown. The effect of sample size appears to be a suppression of fracture initiation given that the modulus and post-peak behaviours are identical.

Figure 6 shows the effect of aspect ratio and confirms the qualitative observation from Figures 1 and 2. Again the initial modulus of the samples was the same. The squat samples saw fracture initiated later than the elongated samples and failure was more catastrophic in the latter. The steeper fall post-peak in the longest samples is likely to be due to the ability of the material to move out of the way of the platens.

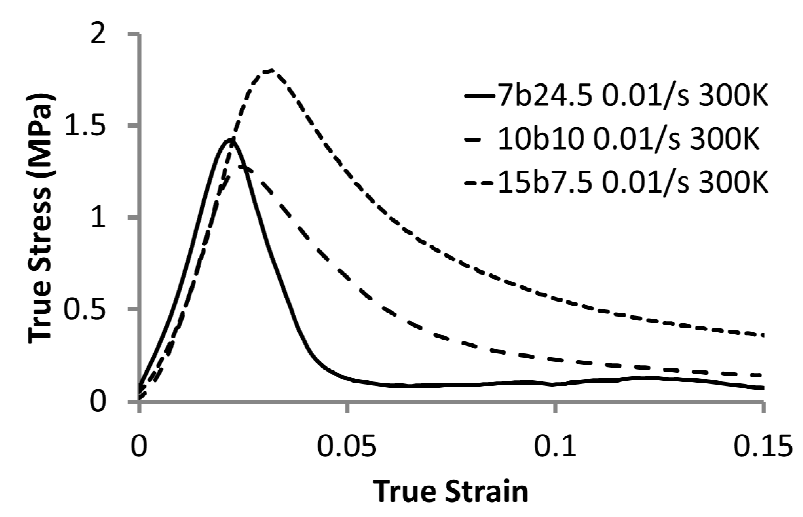

Fig. 6. Effect of aspect ratio on compression behaviour of DPX2 at low rate and room temperature.

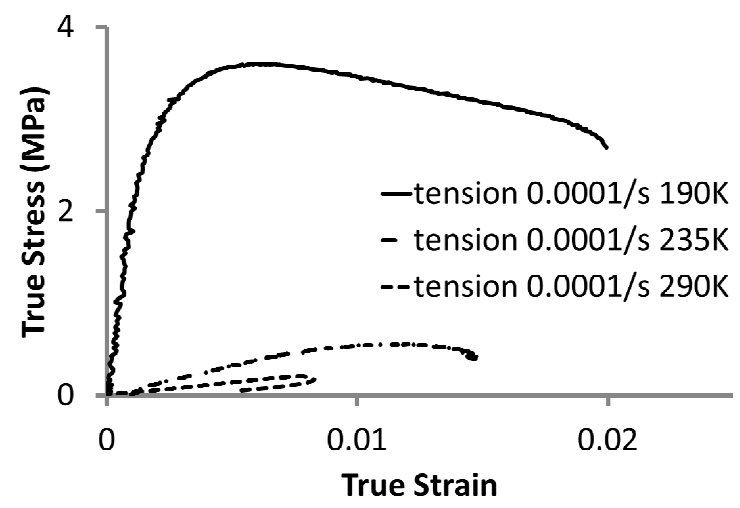

Fig. 7. Effect of temperature on tensile behaviour of DPX2

The effect of temperature on tensile response at low rate is shown in Figure 7 and on compression in Figure 8. The transition from catastrophic failure to a response characterised by composite damage is seen as temperature falls.

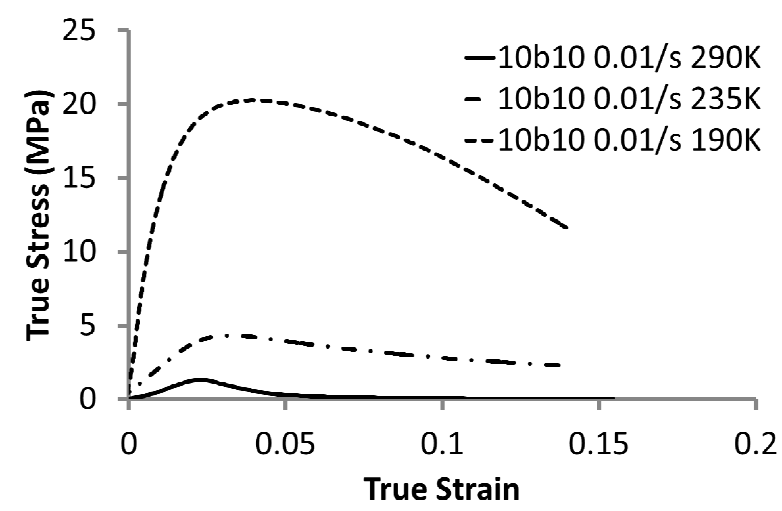

Fig. 8. Effect of temperature on compression behaviour of DPX2.

Gauge traces were converted to stress and strain using the standard SHPB equations. A comparison of the 1-wave and 3-wave analyses is given in Figure 9 and shows that equilibrium is not properly achieved until after the peak strains seen in the low rate tests. It was observed during these tests that the widespread cracking seen at low rate did not occur. This may be due to the size of the specimens but this is counter to the output of 
Figures 5 and 6. An inference is that more debonding is occurring in the samples which may lead to more volume increase than at low rate and this would render the calculated values of stress from the SHPB tests less accurate due to violation of the assumptions used.

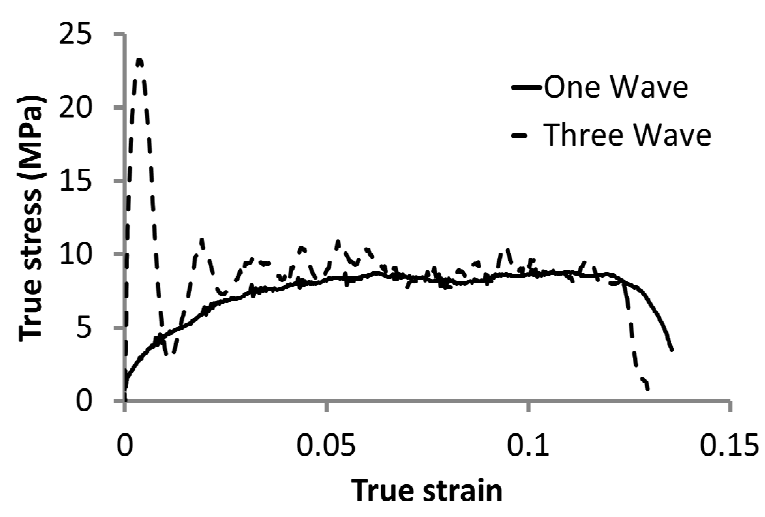

Fig. 9. Comparison of stress vs strain at $300 \mathrm{~K}$ and $1600 / \mathrm{s}$ calculated via 1-wave and 3-wave analyses.

Comparing across all rates used at room temperature in compression, Figure 10 emphasises the change in shape of the response from that dominated by fracture to one of composite damage. he sharp peak is suppressed and resistance to flow is maintained to high strains. Varying temperature does not change the response significantly as is obvious from Figure 11 which shows the highest and lowest temperatures used in the SHPB testing.

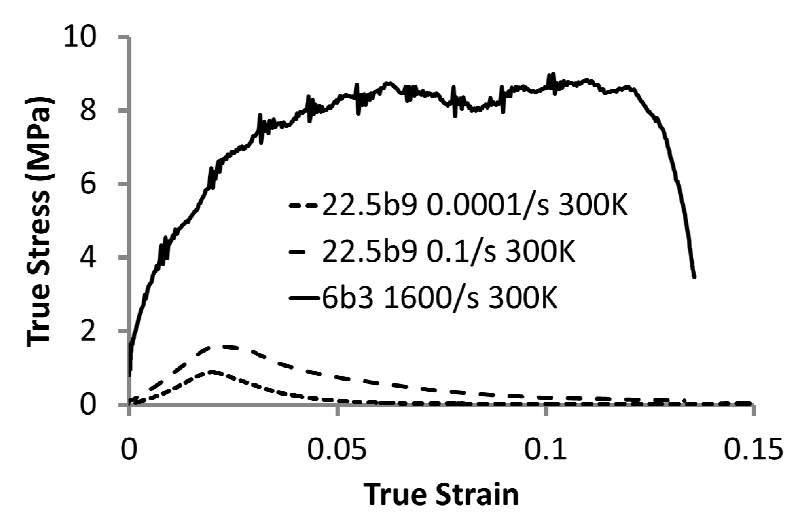

Fig. 10. Comparison of compression response across seven decades of rate at room temperature.

As part of the work some Brazilian disc tests were performed at high rate using the standard disc geometry and rounded anvils placed between the input and output bars of the Hopkinson bar apparatus. The intent was to measure high rate tension in what was supposed to be a low toughness material. The response of the specimen under those conditions was characterised by viscous flow across the specimen. Cracking was totally suppressed and the data could not be used satisfactorily to give quantitative information. Qualitatively, though, the tests supported a link between rate and suppression of cracking/localisation.

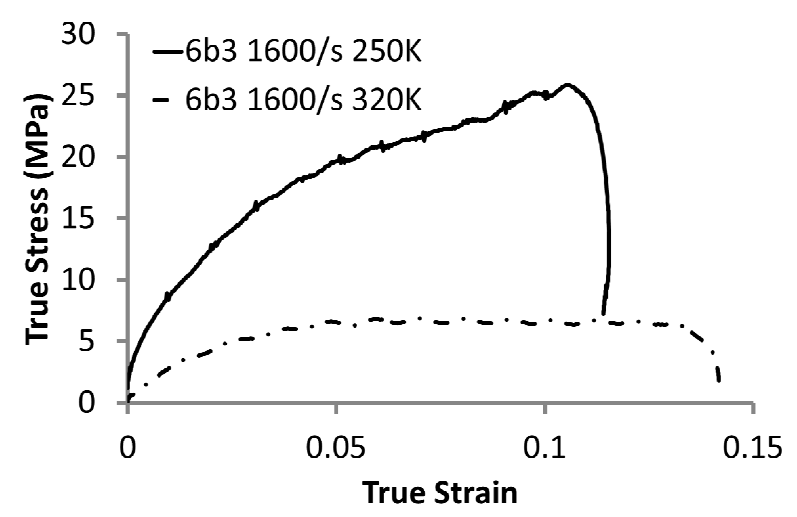

Fig. 11. Upper and lower bounds of temperature response at high rate.

Presuming that a valid modulus can be extracted from the 1-wave analysis at high rates Young's modulus for the whole set of compression data can be compared against the storage modulus data acquired using DMA, Figure 12. A shift factor is applied to the measurement temperature for each of the compression tests to bring them to $1 \mathrm{~Hz}$ equivalent. The equation used is

$$
T^{r}=T+\operatorname{Alog}_{10}\left(\frac{\varepsilon}{\varepsilon_{0}}\right)
$$

where $\dot{\varepsilon}_{0}=1 / \mathrm{s}$ and $\mathrm{A}$ is the shift factor. The fit in Figure 12 has $\mathrm{A}=30 \mathrm{~K}$.

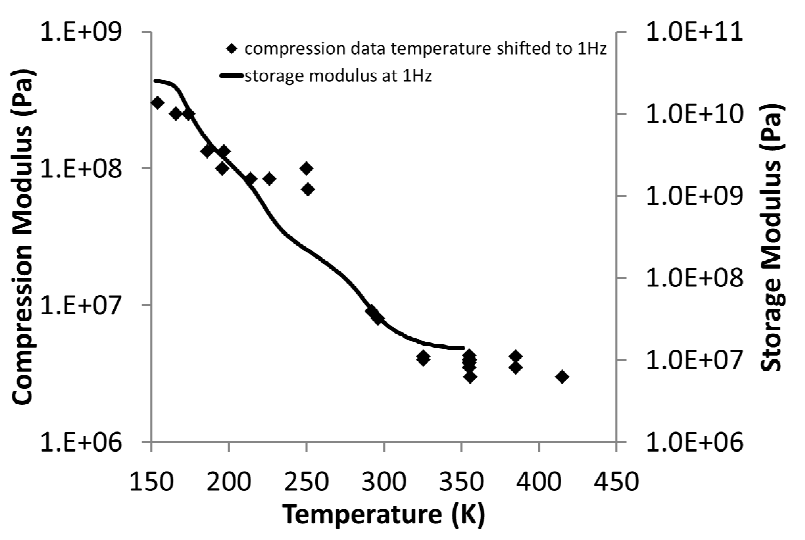

Fig. 12. Comparison between the storage modulus for DPX2 and measured Young's modulus with a shift factor to make the rates equivalent.

Examining Figure 12 brings up some concerns with this approach. The magnitudes of the moduli are radically different and a fit would not be found if they were plotted on the same scales. The high temperature plateau modulus also appears to be different. This is indicating that classical time-temperature superposition is not appropriate. An alternative approach is to find two tests at different rates and temperatures that are equivalent. Such a pair is shown in Figure 13. 


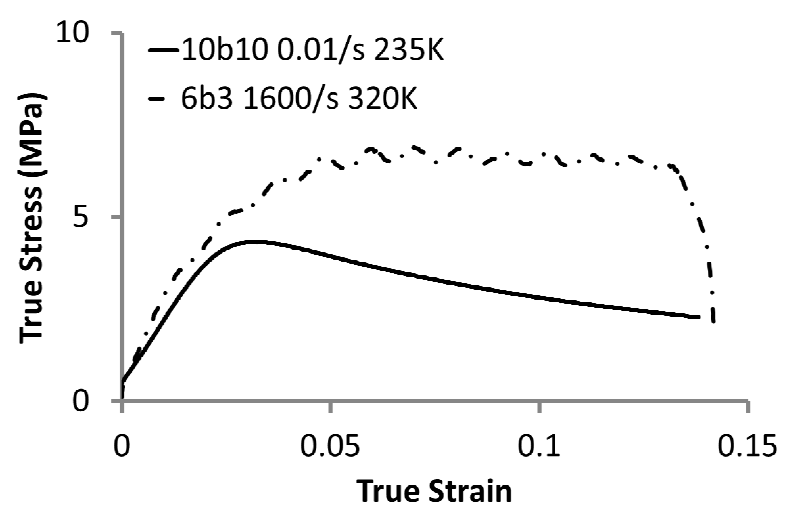

Fig. 13. Two tests carried out under different conditions that have the same Young's modulus.

Whilst the modulus at small strain is the same for both of these tests the difference in damage generation during the tests can be seen from the shape and stress achieved during the tests. With a difference of 5 orders of magnitude in rate and $85 \mathrm{~K}$ in temperature this is equivalent to a shift of $17 \mathrm{~K}$ per decade of rate significantly lower than the shift factor that is derived from Figure 12.

\section{Numerical Simulation}

The compression data was fitted to the physically-based Porter-Gould composite model [2] for use with the QinetiQ version of DYNA 3D. A SESAME-format equation of state was predicted from the properties of the components in DPX2 [3] without reference to data using methods based on Group Interaction Modelling [4]. Verification of the implemented model was demonstrated by comparing simulation of the output pulse with that measured, Figure 14. Inevitably, given that the model was fitted over the full range of strain rate and temperature, there is some inaccuracy in describing the experiment but the overall shape and stress achieved is good.

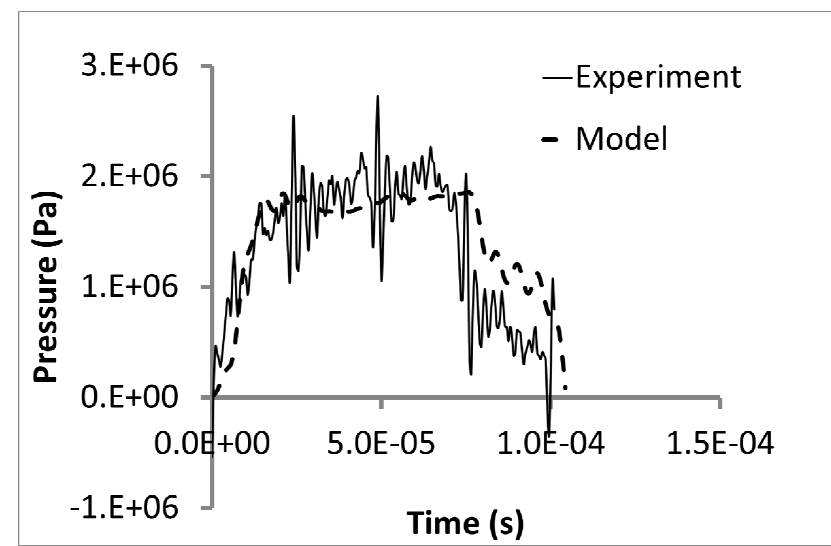

Fig. 14. Comparison of output pulses from simulation and experiment to verify the material models used.

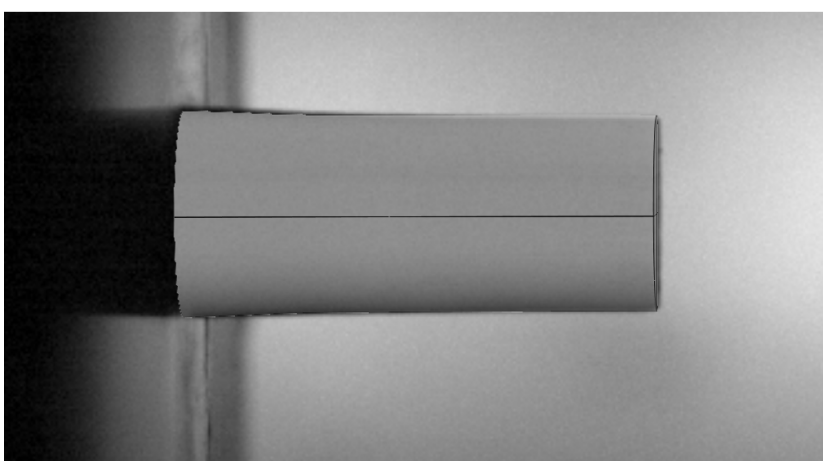

Fig. 15. Comparison between simulation and experiment for a Taylor cylinder launched at $14 \mathrm{~m} / \mathrm{s}$.

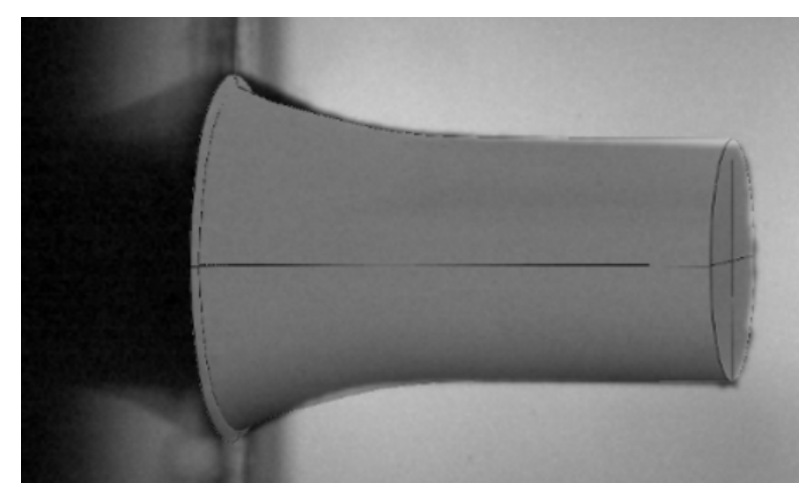

Fig. 16. Comparison between simulation and experiment for a Taylor cylinder launched at $30 \mathrm{~m} / \mathrm{s}$.

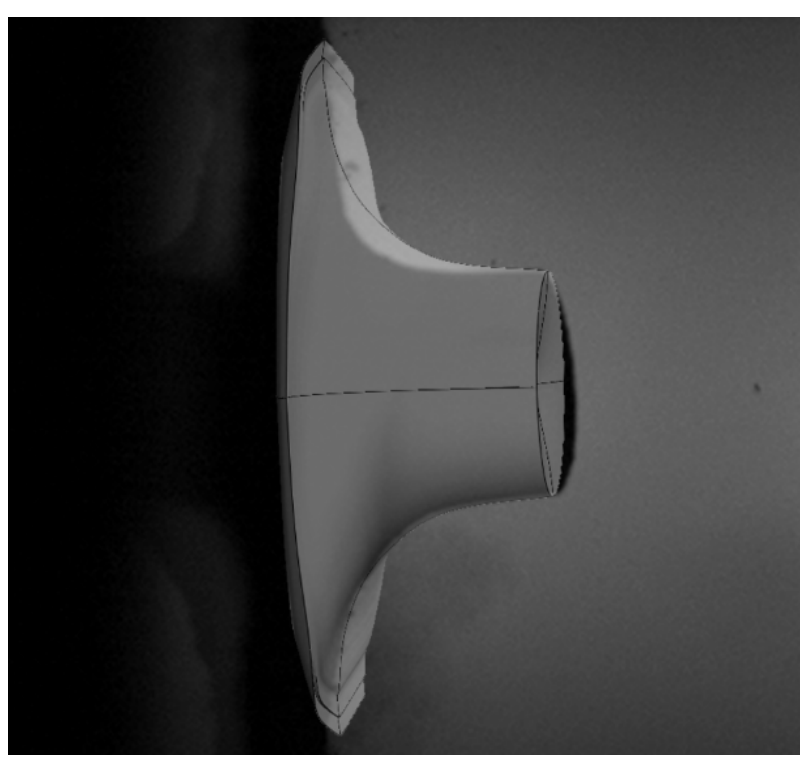

Fig. 17. Comparison between simulation and experiment for a Taylor cylinder launched at $84 \mathrm{~m} / \mathrm{s}$.

The same model was then used to predict the evolution of shape during the three Taylor cylinder experiments. The results are shown in Figures 15, 16 and 17. The shapes predicted by numerical simulation are overlain on the high speed video record and show very good comparison. The frames used were those where the cylinders had come to equilibrium with the anvil just prior to rebounding. The cylinders were recovered intact in all cases. This is especially surprising at the highest 
impact speed given the amount of strain seen in the video.

The excellence of the simulation prediction gives great confidence not only in the Porter-Gould model as a cost-effective route to capability but also in the predicted equation of state.

\section{Conclusions}

A testing programme on DPX2 has gathered a substantial amount of data and has demonstrated the sensitivity of material response to the mode of testing. Fracture at low rate is catastrophic and very similar to granular materials whereas the high rate deformation is dominated by homogeneous gradual damage without significant failure. The reasons behind this change in response are not understood and further analysis of the data should be carried out given the desire to use the material in extreme conditions.

Predictions of material response using numerical simulation have been excellent and give great confidence in their use for design and assessment of systems containing DPX2.

The authors would like to acknowledge funding of this work from UK MOD via WSTC contract DSTLX-1000085224.

\section{References}

1. G.W. Brown, J.A. Tencate, R. DeLuca, P.J. Rae, S.N. Todd, Proceedings of the SEM Annual Conference Albuquerque New Mexico USA, p731 (2009)

2. R. Cornish, D. Porter, P. Church, P. Gould, T. Andrews, B. Proud, D. Drodge, C. Siviour, Shock Compression of Condensed Matter, Hawaii USA, p777 (2007)

3. D. Porter and P.J. Gould, A general equation of state for polymeric materials, Journal De Physique. IV 134, pp373-378 (2006)

4. D. Porter and P.J. Gould, Predictive nonlinear constitutive relations in polymers through loss history, International Journal of Solids and Structures, 46 pp1981-1993 (2009) 